\title{
Call Admission Control Algorithms in OFDM-based Wireless Multiservice Networks
}

\author{
Yan Zhang • Yifan Chen • Jianhua He • \\ Cheng-Xiang Wang • Athanasios V. Vasilakos
}

Published online: 28 June 2008

CC Springer Science+Business Media, LLC. 2008

\begin{abstract}
Orthogonal frequency division multiplexing (OFDM) is becoming a fundamental technology in future generation wireless communications. Call admission control is an effective mechanism to guarantee resilient, efficient, and quality-of-service (QoS) services in wireless mobile networks. In this paper, we present several call admission control algorithms for OFDM-based wireless multiservice networks. Call connection requests are differentiated into narrow-band calls and wide-band calls. For either class of calls, the traffic process is characterized as batch arrival since each call may request multiple subcarriers to satisfy its QoS requirement. The batch size is a random variable following a probability mass function (PMF) with realistically maximum value. In addition, the service times for wide-band and narrow-band calls are different. Following this, we perform a tele-traffic queueing analysis for OFDM-based wireless multiservice networks. The formulae for the significant performance metrics call blocking probability and bandwidth utilization are developed. Numerical investigations are presented to demonstrate the interaction between key parameters and performance metrics. The performance tradeoff among different call admission control algorithms is discussed. Moreover, the analytical model has been validated by simulation. The methodology
\end{abstract}

Y. Zhang (ख)

Simula Research Laboratory, Martin Linges vei 17, Fornebu, P. O. Box 134, 1325 Lysaker, Norway

e-mail: yanzhang@ieee.org

Y. Chen

University of Greenwich, London, UK

e-mail: y.chen@greenwich.ac.uk

J. He

Swansea University, Swansea, UK

e-mail: j.he@swansea.ac.uk

C.-X. Wang

Heriot Watt University, Edinburgh, UK

e-mail: cheng-xiang.wang@hw.ac.uk

A. V. Vasilakos

University of Western Macedonia, Athens, Greece

e-mail: vasilako@ath.forthnet.gr 
as well as the result provides an efficient tool for planning next-generation OFDM-based broadband wireless access systems.

Keywords OFDM - Subcarrier allocation - Call admission control · Call blocking probability · Bandwidth utilization · Queueing system · Wireless multiservice networks

\section{Introduction}

Orthogonal frequency division multiplexing (OFDM) is a digital multi-carrier modulation scheme in which a signal is partitioned into several subchannels at different frequency. OFDM-based systems are able to deliver high data rates, achieve high spectral efficiencies, operate in hostile multipath radio environments, and reduce the power consumption. Due to these advantages, OFDM is becoming a fundamental technology in wireless communications and is a widely adopted multiple access scheme in a number of wireless standards, e.g., IEEE 802.11a/g for wireless local area networks (Wireless LAN), IEEE 802.16a/d/e for wireless metropolitan area networks (Wireless MAN), digital audio broadcasting/digital video broadcasting (DAB/DVB) and satellite radio etc. [1-5].

One of the most significant features of OFDM wireless systems is the flexibility in subcarrier allocation for satisfying various service requirements [6,7]. The studies have focused on specific subcarrier allocation algorithms to accommodate users under some constraints (e.g., [8-10] and the references therein). In contrast, the system analysis and model for OFDM subcarrier allocation is rarely studied. The tele-traffic modeling is significant for designing call admission control mechanisms and also equally important for guaranteeing resilient services subject to fluctuating traffic situations. This is also equally important for service operators implementing and deploying OFDM wireless networks. Recently, the work [7] performed a system queueing model for subcarrier allocation issues in the OFDM-based wireless networks. In this work [7], a single service class is considered. It is believed that supporting multimedia services is an indispensable requirement in future generation wireless networks. Hence, it becomes necessary and significant to investigate the call admission control mechanisms and OFDM subcarrier allocation in wireless multiservice networks, which is however not studied in the literature.

The contributions in the paper include three aspects. Firstly, several call admission control schemes are proposed and studied for OFDM-based wireless systems with multiple services. Secondly, system modeling and analysis will be performed for call admission control mechanisms. The call admission control is characterized as a multi-class multi-server batch arrival queueing system to capture the unique property of the subcarrier allocation problem. The queueing model has the following properties: (1) It has multiple batch arrival processes; (2) the processes have different service times; (3) the batch size is not fixed but supposed as a random variable following a probability mass function; (4) the batch size is not infinite but has a practical maximum value. Either of the properties above could significantly complicate the system dynamics and exhibits different characteristics. Thirdly, extensive numerical examples are presented to demonstrate the performance tradeoff among the proposed schemes. We also show the interaction between the performance metrics and tele-traffic parameters, which is helpful to design OFDM-based wireless multiservice networks.

The rest of the paper is organized as follows. In Sect. 2, we describe the system queueing model. In this section, we also present the multi-dimensional Markov chain and we derive the performance measures. Numerical results are given in Sect. 3, followed by concluding remarks in Sect. 4. 


\section{System Model and Call Admission Control Algorithms}

\subsection{Traffic Model}

Let $C$ denote the number of subcarriers in a cell. Denote $R_{b}$ as the average data rate per subcarrier. The particular value of $R_{b}$ can be calculated from the statistical values of the adaptive modulation coding (AMC) parameters for each subcarrier. As a result, a cell has totally $C R_{b}$ rate resources. In multiservice wireless networks, we categorize the call connections into narrow-band call and wide-band call. An exemplary system can be voice/data integrated wireless networks. For each call, it requests multiple subcarriers to satisfy the data rate transmission requirement. As a result, the call requests can be seen as a batch arrival process. It is noteworthy that, for the narrow-band call, it is reasonable to assume multiple subcarriers instead of a single subcarrier since one subcarrier may not be sufficient to support its data rate request in an OFDM wireless system.

\subsubsection{Batch Blocking Scheme}

Upon the arrival of a narrow-band call, the call may request a number of subcarriers $k$ to satisfy the data rate requirement. If the number of unoccupied subcarriers is smaller than the required number of subcarriers $k$, then the call is blocked. Otherwise, if the number of free subcarriers is equal or greater than the batch size $k$, then the call will be accepted. This policy is also applicable to wide-band calls. That is, upon the moment of a wide-band call arrival, the call may request a number of subcarriers $k$ to satisfy the data rate requirement. If the number of unoccupied subcarriers is smaller than $k$, then the wide-band call is blocked. Otherwise, if the number of free subcarriers is equal or greater than $k$, then the wide-band call will be accepted. This call admission control mechanism is named as batch blocking scheme.

\subsubsection{Partial Blocking Scheme}

When a narrow-band call arrives, and the number of available subcarriers is less than the required number, the call is not blocked but accepted with degraded QoS. In other words, if an arriving narrow-band call requests $k$ subcarriers, while the number of free subcarriers is smaller than the batch size $k$, then the narrow-band call will be accepted with a provided service of these free subcarriers. This policy can also be applied to wide-band calls. This call admission control mechanism is named as partial blocking scheme.

Either the batch blocking scheme or the partial blocking scheme can be applicable to narrow-band calls. Similarly, wide-band calls can employ either the batch blocking scheme or the partial blocking scheme. As a consequence, there are four combinations.

- COMB1: Both the narrow-band and wide-band calls employ the batch blocking scheme

- COMB2: Narrow-band calls employ the batch blocking scheme while wide-band calls use the partial blocking scheme

- COMB3: Narrow-band call employs the partial blocking scheme while wide-band calls use the batch blocking scheme

- COMB4: Both the narrow-band and wide-band calls employ the partial blocking scheme

In the following, we will develop an analytical model and a simulation model to evaluate the trade-off among these different mechanisms. 
The narrow-band call connections are consequently characterized as follows. The narrow-band call requests follow the Poisson process with the mean batch (or group) arrival rate $\lambda_{n}$. At each arrival instant, the number of the batch size is denoted as $x_{n}$. Let the discrete random variable $x_{n}$ follow the probability mass function (PMF) $x_{n, j}\left(j=1,2, \ldots, N_{n}\right)$ where $N_{n} R_{b}$ denotes the highest data rate that a narrow-band call may request. Then,

$$
\sum_{j=1}^{N_{n}} x_{n, j}=1 ; \quad 1 \leq j \leq N_{n} \leq C .
$$

The average value of the group size is given by

$$
\bar{x}_{n}=\sum_{j=1}^{N_{n}} j x_{n, j},
$$

where $\bar{X}$ represents the expected value of the random variable $X$. Equivalently, $\bar{x}_{n} R_{b}$ represents the average data rate that a narrow-band call requests. The service time is exponentially distributed with mean $1 / \mu_{n}$. Here, the exponential distributed service time is not exceptional. The simulation results in Sect. 3 indicate that different distribution functions for the service time lead to insignificant discrepancy on the performance metrics. Hence, the exponential distribution for the service time is able to provide sufficient accuracy.

Similarly, the wide-band call requests follow the Poisson process with the mean batch arrival rate $\lambda_{w}$. At each arrival moment, the number of batch size is denoted as $x_{w}$. Let this discrete random variable $x_{w}$ follow the PMF $x_{w, j}\left(j=1,2, \ldots, N_{w}\right)$ where $N_{w}$ denotes the highest data rate $N_{w} R_{b}$ that a wide-band call may request. Then,

$$
\sum_{j=1}^{N_{w}} x_{w, j}=1 ; \quad 1 \leq j \leq N_{w} \leq C .
$$

The average value of the group size in a wide-band call is given by

$$
\bar{x}_{w}=\sum_{j=1}^{N_{w}} j x_{w, j} .
$$

The service time is exponentially distributed with mean $1 / \mu_{w}$. Hence, the cell has two batch arrival processes. The batch size for each process is a random variable having a maximum value. The service times for the two processes are different. Thereafter, it can be characterized as a multi-class multi-server batch arrival queueing system.

\subsection{COMB1: Both Narrow-band Call and Wide-band Call Use Batch Blocking Scheme}

In this strategy, at the time at which a narrow-band call arrives, if the number of available subcarriers is less than the required number of subcarrier, the call is blocked. In other words, if an arriving narrow-band call requests $k_{n}\left(1 \leq k_{n} \leq N_{n}\right)$ subcarriers, while the number of unoccupied subcarriers is smaller than the batch size $k_{n}$, then the narrow-band call is blocked. This policy is also applicable to wide-band calls. Define the system state as $(i, j)$ with $i$ representing the number of subcarriers used by narrow-band calls and $j$ the number of subcarriers used by wide-band calls. Then, the state space $\Gamma$ is given by

$$
\Gamma=\{(i, j) \mid 0 \leq i \leq C, 0 \leq j \leq C, 0 \leq i+j \leq C\} .
$$




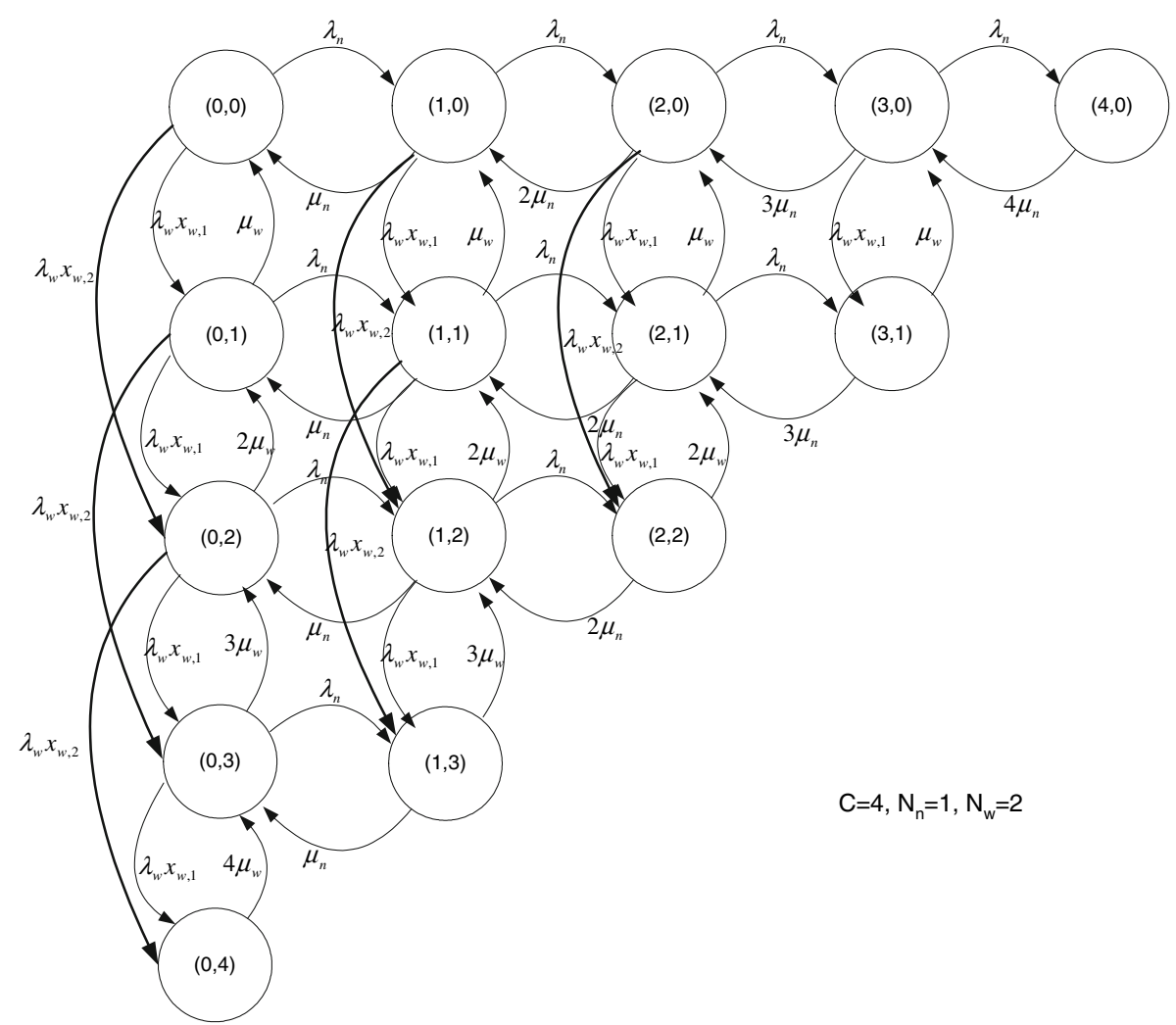

Fig. 1 An example of the transit rate diagram in the COMB1 situation $\left(C=4, N_{n}=1, N_{w}=2\right)$

Figure 1 illustrates an intuitive example when $C=4, N_{n}=1, N_{w}=2$. We take the specific state $(1,2)$ as an example. There are four events that can trigger a state transition from the current state $(1,2)$ :

- Narrow-band call arrival: transit to the neighbouring state $(2,2)$ with rate $\lambda_{n}$.

- Narrow-band call completion: transit to the neighbouring state $(0,2)$ with rate $\mu_{n}$.

- Wide-band call arrival: transit to the neighbouring state $(1,3)$ with rate $\lambda_{w} x_{w, 1}$. Note that the transition to state $(1,4)$ is not feasible, because this state is invalid in the state space $\Gamma$. In addition, if this wide-band call requires two subcarriers with rate $\lambda_{w, 2}$, then the call is blocked without state transition.

- Wide-band call completion: transit to the neighbouring state $(1,1)$ with rate $2 \mu_{w}$.

Following the similar reasoning, we are able to analyze the state transition entering or exiting a particular state; and obtain the transit rate diagram with generalized parameters. Let $\pi(i, j)$ be the steady state probability distribution for a valid state $(i, j) \in \Gamma$. We develop the set of global balance equations. In what follows, $b_{i, j}$ represents the total transit rate out of the state $(i, j) \in \Gamma$.

For the state $(i, j)=(0,0)$,

$$
b_{0,0} \pi(0,0)=\mu_{n} \pi(1,0)+\mu_{w} \pi(0,1)
$$

where $b_{0,0}=\lambda_{n}+\lambda_{w}$. 
For the states $(i, j)$ with $1 \leq i<C$ and $j=0$,

$$
b_{i, 0} \pi(i, 0)=(i+1) \mu_{n} \pi(i+1,0)+\sum_{k=1}^{\min \left(i, N_{n}\right)} x_{n, k} \lambda_{n} \pi(i-k, 0)+\mu_{w} \pi(i, 1)
$$

where

$$
b_{i, 0}=i \mu_{n}+\sum_{k=1}^{\min \left(C-i, N_{n}\right)} x_{n, k} \lambda_{n}+\sum_{k=1}^{\left(C-i, N_{w}\right)} x_{w, k} \lambda_{w} .
$$

For the states $(i, j)$ with $i=0$ and $1 \leq j<C$,

$$
\begin{aligned}
b_{0, j} \pi(0, j)= & (j+1) \mu_{w} \pi(0, j+1) \\
& +\sum_{k=1}^{\min \left(j, N_{w}\right)} x_{w, k} \lambda_{w} \pi(0, j-k)+\mu_{n} \pi(1, j)
\end{aligned}
$$

where

$$
b_{0, j}=j \mu_{w}+\sum_{k=1}^{\min \left(C-j, N_{w}\right)} x_{w, k} \lambda_{w}+\sum_{k=1}^{\left(C-j, N_{n}\right)} x_{n, k} \lambda_{n} .
$$

For the states $(i, j)$ with $0<i+j<C$,

$$
\begin{aligned}
b_{i, j} \pi(i, j)= & (i+1) \mu_{n} \pi(i+1, j)+(j+1) \mu_{w} \pi(i, j+1) \\
& +\sum_{k=1}^{\min \left(i, N_{n}\right)} x_{n, k} \lambda_{n} \pi(i-k, j)+\sum_{k=1}^{\min \left(j, N_{w}\right)} x_{w, k} \lambda_{w} \pi(i, j-k)
\end{aligned}
$$

where

$$
b_{i, j}=i \mu_{n}+j \mu_{w}+\sum_{k=1}^{\min \left(C-i-j, N_{n}\right)} x_{n, k} \lambda_{n}+\sum_{k=1}^{\min \left(C-i-j, N_{w}\right)} x_{w, k} \lambda_{w} .
$$

For the states $(i, j)$ with $i+j=C$,

$$
b_{i, j} \pi(i, j)=\sum_{k=1}^{\min \left(i, N_{n}\right)} x_{n, k} \lambda_{n} \pi(i-k, j)+\sum_{k=1}^{\min \left(j, N_{w}\right)} x_{w, k} \lambda_{w} \pi(i, j-k)
$$

where $b_{i, j}=i \mu_{n}+j \mu_{w}$.

In addition, the summation of all steady state probabilities satisfies the normalization constraint $\sum_{(i, j) \in \Gamma} \pi(i, j)=1$. Combining the equations above, we can solve the set of the linear equations and consequently the steady state probability. In particular, the set of linear equations can be solved by using an iterative method called successive over-relaxation (SOR) $[11,12]$. Then, we can obtain the steady state probability.

Let $P_{n}$ and $P_{w}$ denote the narrow-band and wide-band call blocking probabilities, respectively. At the time when a particular narrow-band (or wide-band) call with batch size $k$ arrives, and the number of available subcarriers is less than $k$, the call is blocked. As a result, the narrow-band call blocking probability is expressed as

$$
P_{n}=\sum_{(i, j) \in \Gamma} \pi(i, j) \sum_{k=C-i-j+1}^{N_{n}} x_{n, k} .
$$


The wide-band call blocking probability is given by

$$
P_{w}=\sum_{(i, j) \in \Gamma} \pi(i, j) \sum_{k=C-i-j+1}^{N_{w}} x_{w, k} .
$$

The bandwidth utilization $\gamma$ is defined as the ratio of the average number of busy subcarriers to the total number of subcarriers $C$, i.e.,

$$
\gamma=\frac{\sum_{(i, j) \in \Gamma}(i+j) \pi(i, j)}{C} .
$$

2.3 COMB2: Narrow-band Call Uses Batch Blocking Scheme while Wide-band Call Use Partial Blocking Scheme

In this mechanism, the narrow-band call follows the batch blocking scheme while the wideband call follows the partial blocking scheme. We develop the set of global balance equations.

For the state $(i, j)=(0,0)$

$$
b_{0,0} \pi(0,0)=\mu_{n} \pi(1,0)+\mu_{w} \pi(0,1)
$$

where $b_{0,0}=\lambda_{n}+\lambda_{w}$.

For the states $(i, j)$ with $1 \leq i<C$ and $j=0$,

$$
b_{i, 0} \pi(i, 0)=(i+1) \mu_{n} \pi(i+1,0)+\sum_{k=1}^{\min \left(i, N_{n}\right)} x_{n, k} \lambda_{n} \pi(i-k, 0)+\mu_{w} \pi(i, 1)
$$

where

$$
b_{i, 0}=i \mu_{n}+\sum_{k=1}^{\min \left(C-i, N_{n}\right)} x_{n, k} \lambda_{n}+\lambda_{w} .
$$

For the states $(i, j)$ with $i=0$ and $1 \leq j<C$,

$$
\begin{aligned}
b_{0, j} \pi(0, j)= & (j+1) \mu_{w} \pi(0, j+1) \\
& +\sum_{k=1}^{\min \left(j, N_{w}\right)} x_{w, k} \lambda_{w} \pi(0, j-k)+\mu_{n} \pi(1, j)
\end{aligned}
$$

where

$$
b_{0, j}=j \mu_{w}+\lambda_{w}+\sum_{k=1}^{\left(C-j, N_{n}\right)} x_{n, k} \lambda_{n} .
$$

For the states $(i, j)$ with $0<i+j<C$,

$$
\begin{aligned}
b_{i, j} \pi(i, j)= & (i+1) \mu_{n} \pi(i+1, j)+(j+1) \mu_{w} \pi(i, j+1) \\
& +\sum_{k=1}^{\min \left(i, N_{n}\right)} x_{n, k} \lambda_{n} \pi(i-k, j)+\sum_{k=1}^{\min \left(j, N_{w}\right)} x_{w, k} \lambda_{w} \pi(i, j-k)
\end{aligned}
$$

where

$$
b_{i, j}=i \mu_{n}+j \mu_{w}+\sum_{k=1}^{\min \left(C-i-j, N_{n}\right)} x_{n, k} \lambda_{n}+\lambda_{w}
$$


For the states $(i, j)$ with $i+j=C$,

$$
b_{i, j} \pi(i, j)=\sum_{k=1}^{\min \left(i, N_{n}\right)} x_{n, k} \lambda_{n} \pi(i-k, j)+\sum_{k=1}^{\min \left(j, N_{w}\right)}\left[\sum_{l=k}^{N_{w}} x_{w, l}\right] \lambda_{w} \pi(i, j-k)
$$

where $b_{i, j}=i \mu_{n}+j \mu_{w}$.

The summation of all steady state probabilities satisfies the normalization constraint. We assume that the system state is $(i, j)$ when a call arrives. The narrow-band call blocking probability is given by

$$
P_{n}=\sum_{(i, j) \in \Gamma} \pi(i, j) \sum_{k=C-i-j+1}^{N_{n}} x_{n, k} .
$$

A wide-band call is blocked when all subcarriers are occupied. Hence, the wide-band call blocking probability is given by

$$
P_{w}=\sum_{(i, j) \in \Gamma \text { and }(i+j)=C} \pi(i, j) .
$$

The bandwidth utilization is given by (14).

2.4 COMB3: Narrow-band Call Uses Partial Blocking Scheme While Wide-band

Call Use Batch Blocking Scheme

In this mechanism, the narrow-band call follows the partial blocking scheme while the wideband call follows the batch blocking scheme. We develop the set of global balance equations.

For the state $(i, j)=(0,0)$,

$$
b_{0,0} \pi(0,0)=\mu_{n} \pi(1,0)+\mu_{w} \pi(0,1)
$$

where $b_{0,0}=\lambda_{n}+\lambda_{w}$.

For the states $(i, j)$ with $1 \leq i<C$ and $j=0$,

$$
b_{i, 0} \pi(i, 0)=(i+1) \mu_{n} \pi(i+1,0)+\sum_{k=1}^{\min \left(i, N_{n}\right)} x_{n, k} \lambda_{n} \pi(i-k, 0)+\mu_{w} \pi(i, 1)
$$

where

$$
b_{i, 0}=i \mu_{n}+\lambda_{n}+\sum_{k=1}^{\left(C-i, N_{w}\right)} x_{w, k} \lambda_{w} .
$$

For the states $(i, j)$ with $i=0$ and $1 \leq j<C$,

$$
\begin{aligned}
b_{0, j} \pi(0, j)= & (j+1) \mu_{w} \pi(0, j+1) \\
& +\sum_{k=1}^{\min \left(j, N_{w}\right)} x_{w, k} \lambda_{w} \pi(0, j-k)+\mu_{n} \pi(1, j)
\end{aligned}
$$


where

$$
b_{0, j}=j \mu_{w}+\sum_{k=1}^{\min \left(C-j, N_{w}\right)} x_{w, k} \lambda_{w}+\lambda_{n}
$$

For the states $(i, j)$ with $0<i+j<C$,

$$
\begin{aligned}
b_{i, j} \pi(i, j)= & (i+1) \mu_{n} \pi(i+1, j)+(j+1) \mu_{w} \pi(i, j+1) \\
& +\sum_{k=1}^{\min \left(i, N_{n}\right)} x_{n, k} \lambda_{n} \pi(i-k, j)+\sum_{k=1}^{\min \left(j, N_{w}\right)} x_{w, k} \lambda_{w} \pi(i, j-k)
\end{aligned}
$$

where

$$
b_{i, j}=i \mu_{n}+j \mu_{w}+\lambda_{n}+\sum_{k=1}^{\min \left(C-i-j, N_{w}\right)} x_{w, k} \lambda_{w} .
$$

For the states $(i, j)$ with $i+j=C$,

$$
b_{i, j} \pi(i, j)=\sum_{k=1}^{\min \left(i, N_{n}\right)}\left[\sum_{l=k}^{N_{n}} x_{n, l}\right] \lambda_{n} \pi(i-k, j)+\sum_{k=1}^{\min \left(j, N_{w}\right)} x_{w, k} \lambda_{w} \pi(i, j-k)
$$

where $b_{i, j}=i \mu_{n}+j \mu_{w}$.

Again, the summation of all steady state probabilities satisfies the normalization constraint. The narrow-band call blocking probability is given by

$$
P_{n}=\sum_{(i, j) \in \Gamma \text { and }(i+j)=C} \pi(i, j) .
$$

The wide-band call blocking probability is given by

$$
P_{w}=\sum_{(i, j) \in \Gamma} \pi(i, j) \sum_{k=C-i-j+1}^{N_{w}} x_{w, k} .
$$

The bandwidth utilization is given by (14).

2.5 COMB4: Both Narrow-band Call and Wide-band Call Use Partial Blocking Scheme

In this strategy, both narrow-band call and wide-band call employ partial blocking scheme. We express the set of global balance equations.

For the state $(i, j)=(0,0)$,

$$
b_{0,0} \pi(0,0)=\mu_{n} \pi(1,0)+\mu_{w} \pi(0,1)
$$

where $b_{0,0}=\lambda_{n}+\lambda_{w}$.

For the states $(i, j)$ with $1 \leq i<C$ and $j=0$,

$$
\begin{aligned}
b_{i, 0} \pi(i, 0)= & (i+1) \mu_{n} \pi(i+1,0)+\mu_{w} \pi(i, 1) \\
& +\sum_{k=1}^{\min \left(i, N_{n}\right)} x_{n, k} \lambda_{n} \pi(i-k, 0)
\end{aligned}
$$

where $b_{i, 0}=i \mu_{n}+\lambda_{n}+\lambda_{w}$. 
For the states $(i, j)$ with $i=0$ and $1 \leq j<C$,

$$
\begin{aligned}
b_{0, j} \pi(0, j)= & (j+1) \mu_{w} \pi(0, j+1)+\mu_{n} \pi(1, j) \\
& +\sum_{k=1}^{\min \left(j, N_{w}\right)} x_{w, k} \lambda_{w} \pi(0, j-k)
\end{aligned}
$$

where $b_{0, j}=j \mu_{w}+\lambda_{n}+\lambda_{w}$.

For the states $(i, j)$ with $0<i+j<C$,

$$
\begin{aligned}
b_{i, j} \pi(i, j)= & (i+1) \mu_{n} \pi(i+1, j)+(j+1) \mu_{w} \pi(i, j+1) \\
& +\sum_{k=1}^{\min \left(i, N_{n}\right)} x_{n, k} \lambda_{n} \pi(i-k, j) \\
& +\sum_{k=1}^{\min \left(j, N_{w}\right)} x_{w, k} \lambda_{w} \pi(i, j-k)
\end{aligned}
$$

where $b_{i, j}=i \mu_{n}+j \mu_{w}+\lambda_{n}+\lambda{ }_{w}$.

For the states $(i, j)$ with $i+j=C$,

$$
\begin{aligned}
b_{i, j} \pi(i, j)= & \sum_{k=1}^{\min \left(i, N_{n}\right)}\left[\sum_{l=k}^{N_{n}} x_{n, l}\right] \lambda_{n} \pi(i-k, j) \\
& +\sum_{k=1}^{\min \left(j, N_{w}\right)}\left[\sum_{l=k}^{N_{w}} x_{w, l}\right] \lambda_{w} \pi(i, j-k)
\end{aligned}
$$

where $b_{i, j}=i \mu_{n}+j \mu_{w}$.

The narrow-band call and wide-band call blocking probabilities are expressed as

$$
P_{n}=P_{w}=\sum_{(i, j) \in \Gamma \text { and }(i+j)=C} \pi(i, j) .
$$

The bandwidth utilization is given by (14).

\section{Numerical Examples}

In this section, we will validate the presented analysis via simulations. In addition, illustrative numerical examples are presented to indicate the interaction between the performance metrics and key parameters. Here, the performance metrics refer to the call blocking probability and bandwidth utilization. The key parameters include the traffic load and service time distribution functions. Following the similar settings in [2], the downlink is based on a multiple-input and multiple-output (MIMO) OFDM air interface. The average coding rate is $2 / 3$ with 16-QAM and spatial multiplexing mode. There are two transmit antennas and up to four receive antennas. The system has a bandwidth of $5 \mathrm{MHz}$ and consists of $C=32$ subcarriers. Based on the settings, the peak throughput is 20.6 Mbps [2]. As a consequence, each subcarrier has an average data rate $R_{b}=20.6 \mathrm{Mbps} / 32=659.2 \mathrm{kbps}$. To validate the analytical model, we have developed a discrete event simulation program in $\mathrm{C}++$.

Figure 2 shows the performance metrics in terms of narrow-band call traffic intensity, which is defined as $\rho_{n}=\bar{x}_{n} \lambda_{n} /\left(C \mu_{n}\right)$. The simulation results (indicated by a symbol over each line) are presented for the purpose of validation. It is observed that the analysis and the 

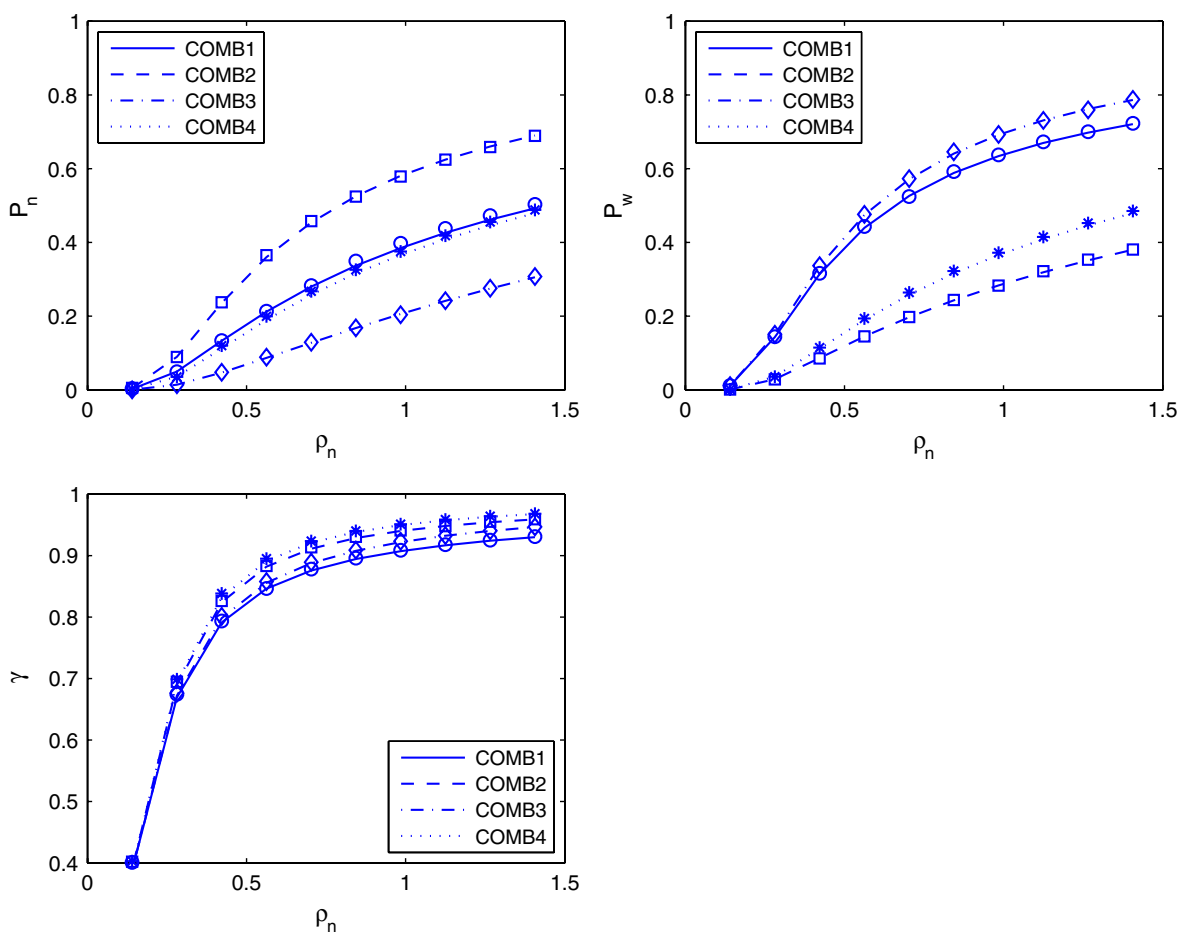

Fig. 2 Performance metrics in terms of narrow-band call traffic intensity. The symbol in each line represents the simulation result. $\left(N_{n}=4, N_{w}=8,1 / \mu_{n}=1 / \mu_{w}=180.0\right)$

simulation match up with each other very well in all four situations. In addition, the three performance metrics increase with the higher traffic intensity $\rho_{n}$, which is intuitively understandable. Comparing the four call admission control algorithms, we can observe that COMB3 is able to achieve the lowest narrow-band call blocking probability while COMB2 achieves the lowest wide-band call blocking probability. With respect to the bandwidth utilization, the difference between COMB2 and COMB3 is very small. Hence, if narrow-band calls demand lower call blocking probability than wide-band calls, the algorithm COMB3 shall be used. Otherwise, COMB2 can be employed. On the other hand, if both narrow-band calls and wide-band calls have similar requirement on call blocking probability, the scheme COMB4 may be a choice, which is also able to achieve maximum bandwidth utilization in these algorithms.

Figure 3 shows the performance metrics in terms of narrow-band call traffic intensity. Different from the previous example, the service times for the narrow-band and wide-band calls are different. The symbol in the figure represents the simulation result. Again, the analysis and the simulation agree with each other. With greater wide-band call traffic intensity, the call blocking probability and bandwidth utilization in Fig. 3 are respectively higher than the results in Fig. 2. Moreover, the similar performance tradeoff can be observed among the four call admission control algorithms.

Figure 4 shows the performance metrics with different service time distributions in the scheme COMB1. In each figure, the exponential distribution, Erlang distribution and hyper- 

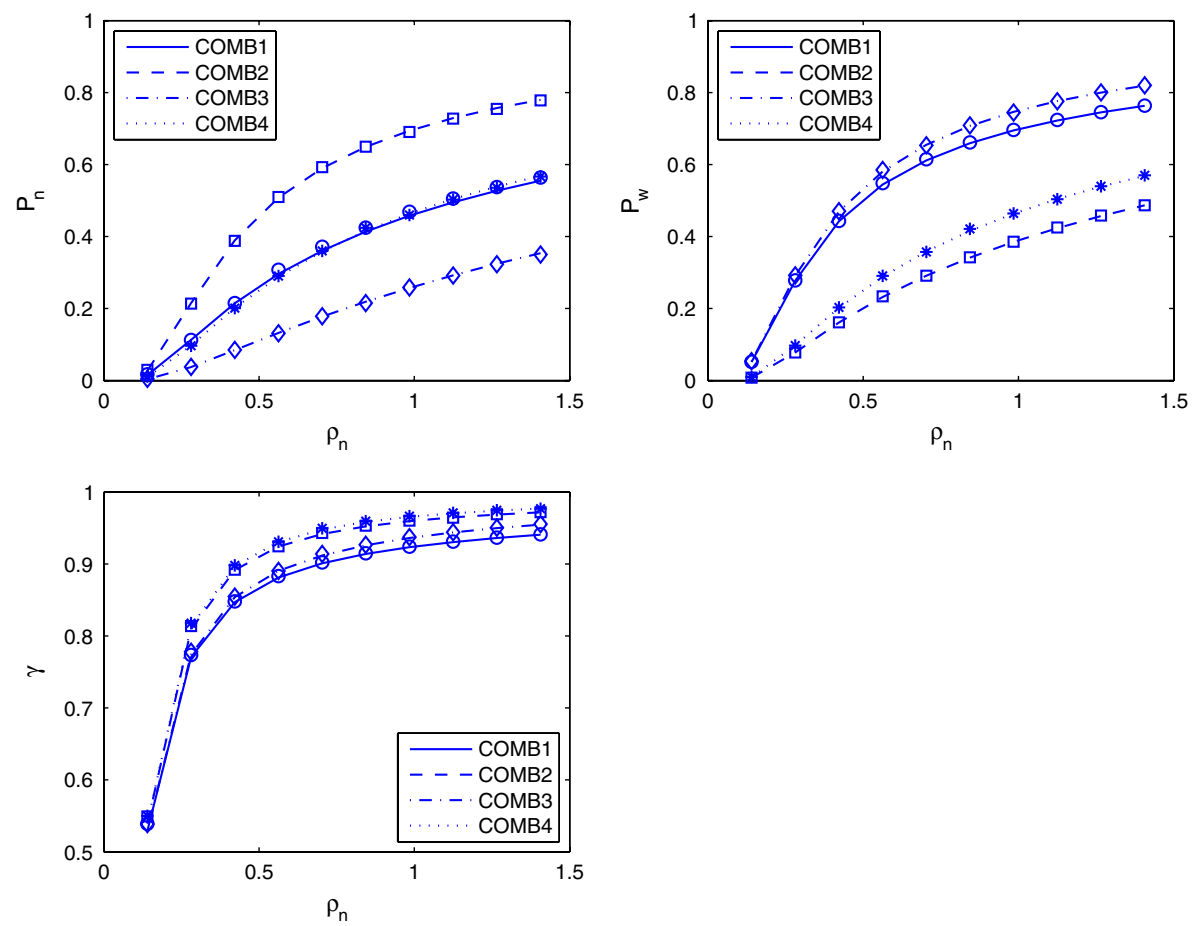

Fig. 3 Performance metrics in terms of narrow-band call traffic intensity. The symbol in each line represents the simulation result. $\left(N_{n}=4, N_{w}=8,1 / \mu_{n}=180.0,1 / \mu_{w}=300.0\right)$

Erlang distribution for the narrow-band and wide-band call service times are employed for comparison. The hyper-Erlang distributed service times have the probability density function

$$
0.4 \times 0.8 \mu_{k} e^{-0.8 \mu_{k} t}+0.6 \times\left(2 \times 1.2 \mu_{k}\right)^{2} t e^{-2 \times 1.2 \mu_{k} t}, k \in\{n, w\} .
$$

Here, a hyper-Erlang distributed service time is employed since a hyper-Erlang distribution has been proven to be able to arbitrarily approximate to the distribution of any positive random variable as well as measured data [13-15]. The comparison indicates that the discrepancy is very small when employing different service time distributions to evaluate the network performance. Hence, adopting the exponential distribution for the service times in the queueing model enables to provide accurate results. It is noteworthy that we have examined the service time distribution effect in the schemes COMB2, COMB3 and COMB4. Similarly, ignorable difference can be observed when we use different service time distributions.

\section{Conclusions}

In this paper, four call admission control algorithms are proposed for OFDM-based wireless multiservice networks. Analytical models are presented to investigate the performance tradeoff among these schemes. The good agreement between the analysis and simulation results under various scenarios validates the correctness of the analytical queueing model. The result indicates that the exponential distribution can provide sufficient accuracy for the 

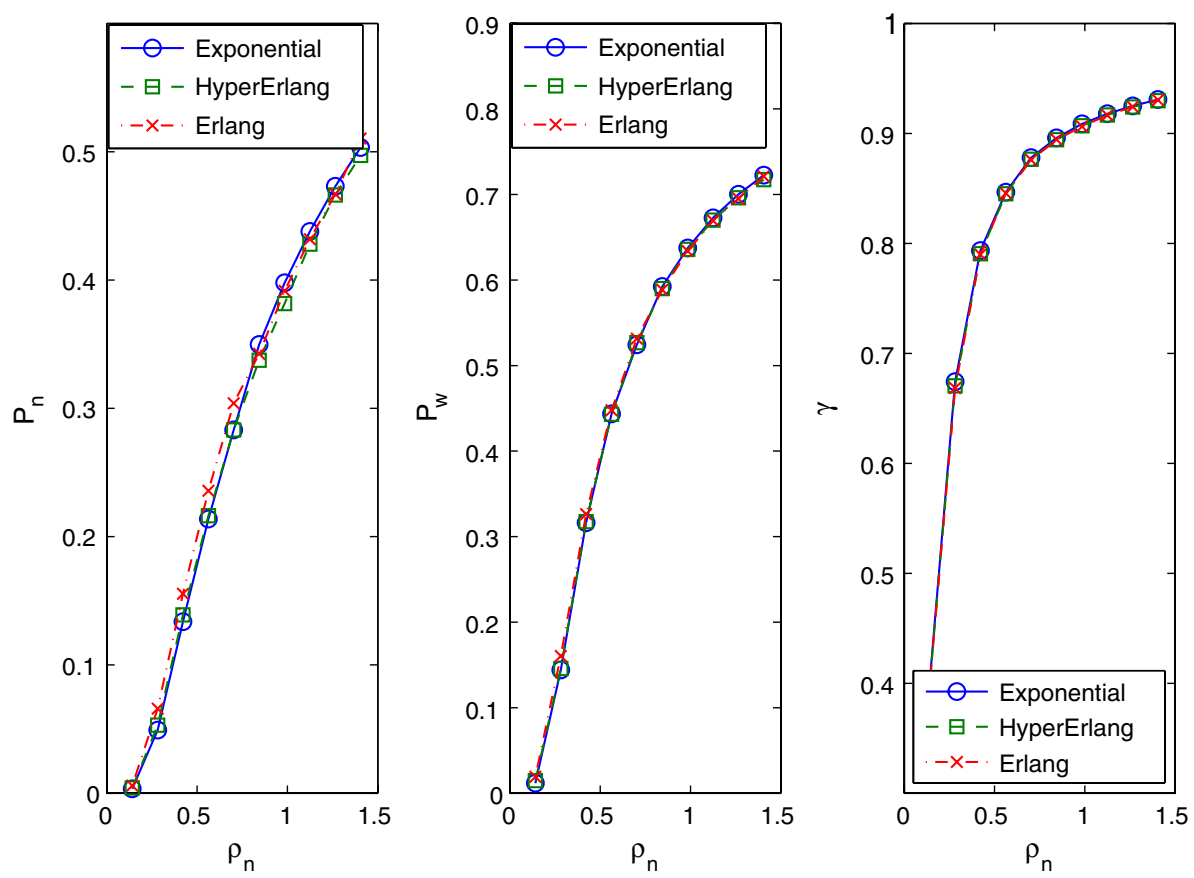

Fig. 4 Performance metrics in terms of narrow-band call traffic intensity $\rho_{n}$ with different service time distribution functions in COMB1

service time in evaluating the call admission control policies. The methodology as well as the result provide an efficient tool for building future generation OFDM-based wireless multiservice networks.

\section{References}

1. Jamalipour, A., Wada, T., \& Yamazato, T. (2005). A tutorial on multiple access technologies for beyond $3 \mathrm{G}$ mobile networks. IEEE Communications Magazine, 43, 110-117.

2. Dubuc, C., Starks, D., Creasy, T., \& Hou, Y. (2004). A MIMO-OFDM prototype for next-generation wireless WANs. IEEE Communications Magazine, 42, 82-87.

3. Bolcskei, H. (2006). MIMO-OFDM wireless systems: basics, perspectives, and challenges. IEEE Wireless Communications, 13(4), 31-37.

4. Ping, L., Guo, Q., \& Tong, J. (2007). The OFDM-IDMA approach to wireless communication systems. IEEE Wireless Communications, 14(3), 18-24.

5. Koffman, I., \& Roman, V. (2002). Broadband wireless access solutions based on OFDM access in IEEE 802.16. IEEE Communications Magazine, 40(4), 96-103.

6. Wong, C. Y., Cheng, R. S., Letaief, K. B., \& Murch, R. D. (1999). Multiuser OFDM with adaptive subcarrier, bit and power allocation. IEEE Journal on Selected Areas in Communications, 17, 1747-1757.

7. Chen, J.-C., \& Chen, W.-S. E. (2006). Call blocking probability and bandwidth utilization of OFDM subcarrier allocation in next-generation wireless networks. IEEE Communications Letters, 10(2).

8. Kivanc, D., \& Lui, H. (2000). Subcarrier allocation and power control for OFDMA. In Proceedings of the 34th Asilomar Conference on Signals, Systems and Computers, Vol. 1, pp. 147-151, 2000.

9. Song, P., \& Cai, L. (2004). Multi-user subcarrier allocation with minimum rate requests for downlink OFDM packet transmission. In Proceedings IEEE VTC'04, Vol. 4, pp. 1920-1924, May 2004.

10. Zhang, Y. J., \& Letaief, K. B. (2005). An efficient resource-allocation scheme for spatial multiuser access in MIMO/OFDM systems. IEEE Transactions on Communications, 53, 107-116. 
11. Cooper, R. B. (1981). Introduction to queueing theory (2nd ed.). New York: North-Holland.

12. Gross, D., \& Harris, C. M. (1974). Fundamentals of queueing theory. New York: Wiley.

13. Fang, Y. (2001). Hyper-Erlang distribution model and its application in wireless mobile networks. $A C M$ Wireless Networks, 7(3), 211-219.

14. Marsan, M. A., Ginella, G., Maglione, R., \& Meo, M. (2004). Performance analysis of hierarchical cellular networks with generally distributed call holding times and dwell times. IEEE Transactions on Wireless Communications, 3(1), 248-257.

15. Kleinrock, L. (1975). Queueing systems. New York, NY: Wiley.

\section{Author Biographies}

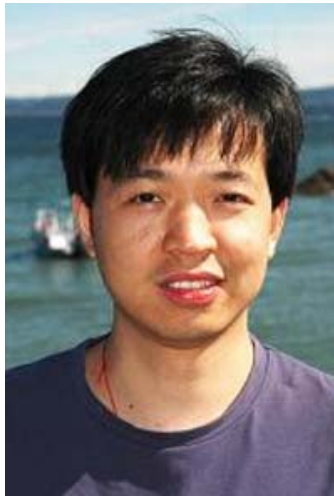

Yan Zhang received PhD degree in School of Electrical \& Electronics Engineering, Nanyang Technological University, Singapore. He is associate editor or on the editorial board of Security and Communication Networks (Wiley), International Journal of Network Security, Transactions on Internet and Information Systems (TIIS), International Journal of Autonomous and Adaptive Communications Systems (IJAACS), International Journal of Ubiquitous Computing and International Journal of Smart Home (IJSH). He is currently serving the Book Series Editor for the book series on "Wireless Networks and Mobile Communications" (Auerbach Publications, CRC Press, Taylor and Francis Group). He has served as co-editor for several books. He serves as organizing committee chairs and technical program committee for many international conferences. He received the Best Paper Award in the IEEE 21st International Conference on Advanced Information Networking and Applications (IEEE AINA-07). From August 2006, he is working with Simula Research Laboratory, Norway. His research interests include resource, mobility, spectrum, data, energy, and security management in wireless networks and mobile computing. He is a member of IEEE and IEEE ComSoc.

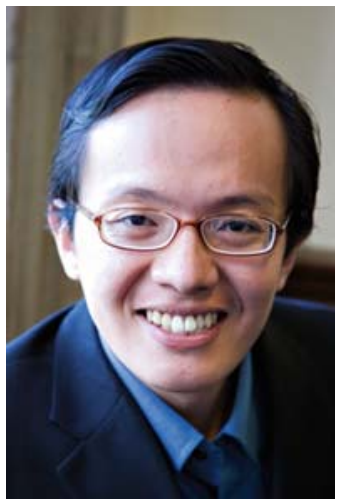

Yifan Chen received the B.Eng (Hons I) and Ph.D degrees in electrical and electronic engineering from Nanyang Technological University, Singapore, in 2002 and 2006, respectively. He is presently with the School of Engineering, University of Greenwich, UK as a lecturer. He is also with the School of Computer, Electronic and Information, Guangxi University, China as an adjunct associate professor. His current research interests involve ultra-wideband radar systems for healthcare applications including microwave imaging of human tissues and non-contact vital-signs monitoring, statistical modeling of mobile radio channels, and wireless communications and geolocation systems. 


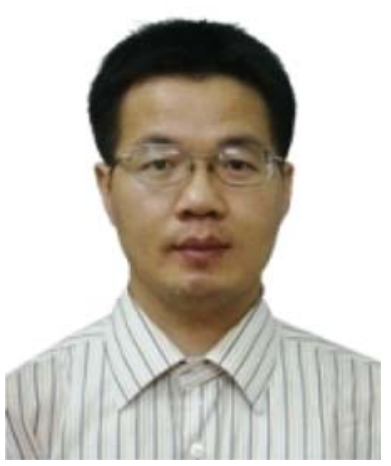

Jianhua He is lecturer in Institute of Advanced Telecommunications, Swansea University, UK. He received his BEng and MEng both in electronics engineering from Huazhong University of Science and Technology (HUST), China, and his $\mathrm{PhD}$ in communications engineering from Nanyang Technology University, Singapore. He joined HUST as an Associate Professor in 2001. From 2004 to 2006, he was with the Department of Electronic \& Electrical Engineering, University of Bristol, UK. He was with Department of Computing \& Electronic Systems, University of Essex, UK from 2006 to 2007. His research interests include medium access control and networking for heterogeneous wireless networks (WiFi, WiMAX, WPAN, WCDMA, sensor networks, mesh networks and mobile ad hoc networks), dynamic radio resource management and spectrum access, cross-layer performance evaluation and optimization for third generation $(3 \mathrm{G})$ mobile communication systems and beyond. He has published 60 journal and conference papers. He serves on the editorial board of Wiley International Journal of Wireless

Communication and Mobile Computing (WCMC), KSII Transactions on Internet and Information Systems, and serves as a guest editor for International Journal of Autonomous and Adaptive Communications Systems. $\mathrm{He}$ is a chair or TPC member of many international conferences. He is a Member of IEEE.

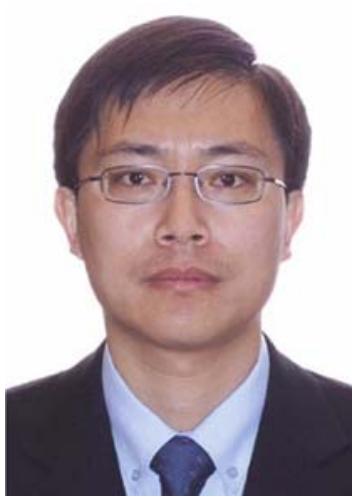

Cheng-Xiang Wang received the BSc and M.Eng degrees in Communication and Information Systems from Shandong University, Shandong, P. R. China, in 1997 and 2000, respectively, and the PhD degree in Wireless Communications from Aalborg University, Aalborg, Denmark, in 2004. Dr Wang has been a Lecturer in Mobile Communications and Networks at Heriot-Watt University, Edinburgh, UK since 2005. He is also an Honorary Fellow of the University of Edinburgh, a Guest Researcher of Xidian University, and an Adjunct Professor of Guilin University of Electronic Technology, P. R. China. From 2001 to 2005, he was a Research Fellow of Mobile Communications with the University of Agder, Norway. From January to April 2004, he was a Visiting Researcher at Baseband Algorithms \& Standardization Lab of Siemens AG-Mobile Phones, Munich, Germany. From 2000 to 2001, he was a Research Assistant with Technical University of HamburgHarburg, Hamburg, Germany. His current research interests include wirless channel modelling and simulation, error models, cognitive radio, mobile-to-mobile communications, cooperative (relay) communications, cross-layer design of wireless networks, MIMO, OFDM, UWB, mobile ad hoc and wireless sensor networks, and 3GPP long term evolution. He has published over 80 papers in refereed journals and conference proceedings. Dr Wang serves as an Editor for four international journals: IEEE Transactions on Wireless Communications, Wireless Communications and Mobile Computing Journal (John Wiley \& Sons), Security and Communication Networks Journal (John Wiley \& Sons), and Journal of Computer Systems, Networks, and Communications (Hindawi). He also serves as a Guest Editor for Special Issue on "Cognitive Radio Systems" of International Journal of Autonomous and Adaptive Communications Systems (Inderscience), TPC vice-chair for IEEE HPCC 2008, TPC track chair for IEEE ICIEA 2008, and TPC member for 25 major international conferences including IEEE VTC 2005-Fall, GLOBECOM 2006, ICC 2007, ICC 2008, GLOBECOM 2008, and ICC 2009. Dr Wang is listed in Who's Who in the World 2008. He is a member of the IEEE and IET and a Fellow of the HEA. 


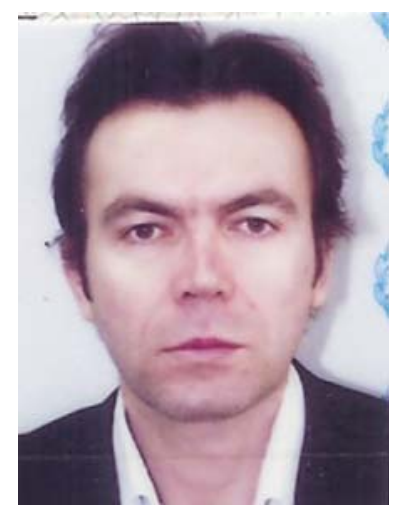

Athanasios V. Vasilakos is currently Professor at the Dept. of Computer and Telecommunications Engineering, University of Western Macedonia, Greece, and Professor at the Graduate Programm of the Dept. of Electrical and Computer Engineering, National Technical University of Athens(NTUA). He is coauthor (withW.Pedrycz) of the books Computational Intelligence in Telecommunications Networks (CRC press, USA, 2001), Ambient Intelligence, Wireless Networking, Ubiquitous Computing (Artech House, USA, 2006), and coauthor (with M.Parashar, S.Karnouskos, W.Pedrycz) Autonomic Communications (Springer, to appear), Arts and Technologies (MIT Press, to appear), Game Theory in Communication Systems(IGI Inc., to appear). He has published more than 150 articles in top international journals and conferences in the area of Machine Learning, Computational Intelligence(CI), CI in Telecommunications Networks, Game Theory, Evolutionary Game Theory, Networking, Mobile Computing and Wireless Networking, Web Services, CI in Bioinformatics, Web-based Education, and, Internet Arts, Interactive Theatre, Visual Dance. He is the Editor-in-chief of the Inderscience Publishers journals: International Journal of Adaptive and Autonomous Communications Systems(IJAACS, http://www.inderscience.com/ijaacs), International Journal of Arts and Technology(IJART, http://www.inderscience.com/ijart).He was or he is at the editorial board of several international journals: Computer Communications (Elsevier, 1988-1991), ACM Applied Computing Reviews(ACM ACR), IEEE Communications Magazine (1999-2002 and 2008-), IEEE Transactions on Systems, Man and Cybernetics(SMC, Part B), IEEE Transactions on Wireless Communications(invited 2008), ACM/Springer Wireless Networks(2008-), Wireless Communications and Mobile Computing(Wiley, 2008-), Soft Computing (Springer), Information Sciences (Elsevier), International Journal on Computational Intelligence Research, International Journal on Cognitive Informatics and Natural Intelligence(IJCiNi), International Journal of Ad Hoc and Ubiquitous Computing(IJAHUC), ACM Computers in Entertainment(ACM CIE), Journal of Computational Intelligencce in Bioinformatics and Systems Biology(JCIBSB), International Journal of Functional Informatics and Personalised Medicine((IJFIPM), International Journal of Mobile Communications (IJMC), International Journal of Computational Science(IJCS), International Journal of Internet Protocol Technology(IJIPT), Cluster Computing(Springer), EURASIP journal on Wireless and Communications Netwoks(WCN), Security and Communications journal(Wiley), Journal of Sensors(Hindawi), Journal of Supercomputing(Springer), Telecommunications Systems(Springer). Guest Editor of several journals, such as IEEE T-Systems, Man and Cybernetics(special issue: Computational Intelligence in Telecommunications Networks, 2003), ACM Transactions on Autonomous and Adaptive Systems (http://www.acm.org/pubs/taas/ special issues on I) Autonomic Communications II) Ambient Intelligence), IEEE Journal on Selected Areas in Communications-IEEE JSAC( Wireless and Pervasive Networks in Healthcare), Artificial Intelligence(special issue: Game Theory) etc. He was chairman of theTelecommunications Committte of the ERUDIT, European network of excellence in fuzzy logic and its applications and he is chairman of the Telecommunications Task Force of the Intelligent Systems Applications Technical Committee (ISATC) of the IEEE Computational Intelligence Society (CIS). Senior Deputy Secretary-General and fellow member of ISIBM www.isibm. $\operatorname{org}$ (International Society of Intelligent Biological Medicine (ISIBM). He is member of the IEEE and ACM. 